\title{
PAPER \\ Congestion-Adaptive and Deadline-Aware Scheduling for Connected Car Services over Mobile Networks
}

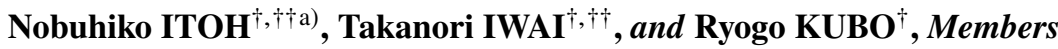

\begin{abstract}
SUMMARY Road traffic collisions are an extremely serious and often fatal issue. One promising approach to mitigate such collisions is the use of connected car services that share road traffic information obtained from vehicles and cameras over mobile networks. In connected car services, it is important for data chunks to arrive at a destination node within a certain deadline constraint. In this paper, we define a flow from a vehicle (or camera) to the same vehicle (or camera) via an MEC server, as a mission critical (MC) flow, and call a deadline of the MC flow the MC deadline. Our research objective is to achieve a higher arrival ratio within the MC deadline for the MC flow that passes through both the radio uplink and downlink. We previously developed a deadline-aware scheduler with consideration for quality fluctuation (DAS-QF) that considers chunk size and a certain deadline constraint in addition to radio quality and utilizes these to prioritize users such that the deadline constraints are met. However, this DAS-QF does not consider that the congestion levels of evolved NodeB (eNB) differ depending on the eNB location, or that the uplink congestion level differs from the downlink congestion level in the same eNB. Therefore, in the DAS-QF, some data chunks of a MC flow are discarded in the eNB when they exceed either the uplink or downlink deadline in congestion, even if they do not exceed the MC deadline. In this paper, to reduce the eNB packet drop probability due to exceeding either the uplink and downlink deadline, we propose a deadline coordination function (DCF) that adaptively sets each of the uplink and downlink deadlines for the MC flow according to the congestion level of each link. Simulation results show that the DAS-QF with DCF offers higher arrival ratios within the MC deadline compared to DAS-QF on its own

key words: QoS, deadline, IoT, connected car, mobile network, scheduling, $M E C$
\end{abstract}

\section{Introduction}

Road traffic collisions are an extremely serious issue worldwide. According to the World Health Organization (WHO), the number of road traffic deaths has now grown to 1.25 million per year [1]. In Japan, where drivers keep to the left side of the road, the number of fatalities is 4,000 per year, and road traffic collisions occurring in blind spots, such as right-turn collisions and rear-end collisions, account for $74 \%$ of all accidents [2]. In an attempt to decrease the occurrence of road traffic collisions, driving safety systems with sensors such as vehicle-mounted cameras and vehicle radar for detecting pedestrians and vehicles have been deployed. However,

\footnotetext{
Manuscript received December 19, 2019.

Manuscript revised March 24, 2020.

Manuscript publicized April 21, 2020.

†The authors are with the Department of Electronics and Electrical Engineering, Keio University, Yokohama-shi, 223-8522

${ }^{\dagger}$ The authors are with the System Platform Research Laboratories, NEC Corporation, Kawasaki-shi, 211-8666 Japan.

a)E-mail: n-itoh@nec.com

DOI: 10.1587/transcom.2019EBT0007
} Japan. these systems cannot detect vehicles and pedestrians in blind spots such as sharp bends and blind intersections.

To mitigate road traffic collisions at intersections, connected cameras are allocated around the intersection. Mobile networks such as long-term evolution (LTE) networks have attracted a great deal of attention as platforms for detecting vehicles and pedestrians in the intersection [3]-[5]. By using mobile networks, a connected car service can collect realtime information such as the image around the intersection and the locations of vehicles from connected devices such as road side cameras and vehicles. The real-time information helps drivers to avoid road traffic collisions. For example, a road traffic collision avoidance scheme with road-side cameras has been proposed in [6]. In London [7] and Beijing [8], many cameras have been deployed for surveillance in public spaces. In the future, many cameras located in public spaces may be utilized for vehicle traffic control.

To guarantee effective connected car services, it is important to deliver a data block within a certain maximum tolerable delay (called a deadline in this work). The Third Generation Partnership Project (3GPP) stipulates that this deadline of an urban intersection is $100 \mathrm{~ms}$ and that the arrival ratio within the deadline is more than $95 \%$ [9]. In this paper, we define a whole data block as a chunk. The arrival ratio within the deadline is calculated as the ratio of the total number of chunks that user equipments (UEs) received before the deadline to the total number of chunks that the UEs sent.

Multi-access edge computing (MEC) is an effective way to provide low-latency services because the MEC server is allocated nearby an evolved NodeB (eNB). Delay-sensitive applications such as road traffic collision avoidance are operated from the MEC server [10]-[14]. By using the MEC server, the distance between vehicles or cameras and the application server can be shorter than when using a mobile network without one. It is important to ensure low latency in the radio section between UEs, such as vehicles and cameras, and eNB. The rapid increase in Internet of Things (IoT) devices has caused growth in data traffic demands [15], and scarcity in last-one-mile bandwidth remains a huge issue across mobile networks [16].

Deadline-aware schedulers have been implemented in eNB to achieve low latency in the last-one-mile radio section [17]-[22]. In the earliest deadline first (EDF) algorithm [17], a data stream with a fast-approaching deadline tends to be prioritized over data streams with a lot of time left before their deadlines. Payload-size and deadline-aware (PayDA) 
is an EDF-based scheduler that performs priority control considering not only the remaining time to the deadline but also the remaining data amount of each data stream [18]. Andreozzi et al. proposed a method considering the deadline and radio quality [19]. Another scheduler, the channeldependent earliest deadline due (CD-EDD) [20], considers wireless channel quality, but does not consider chunk size. In an earlier study, we proposed an EDF-based scheduler that considers the remaining data amount of each data stream and radio quality in addition to the remaining time to deadline [21], [22]. The scheduler [21], called the deadline-aware scheduler with consideration for quality fluctuation (DAS$\mathrm{QF}$ ) in this paper, can increase the arrival ratio within a certain deadline constraint. However, neither the DAS-QF nor the other deadline-aware schedulers described above can be adaptively set to each of the uplink and downlink deadlines for a flow that passes through both the radio uplink and downlink.

Our research objective is to achieve a higher arrival ratio within the deadline for a flow that passes through both the radio uplink and downlink. Ideally, we envision a connected camera that periodically sends an image around an intersection to an MEC server. The MEC server replies with a message for controlling the camera. We assume that the message for controlling the camera includes camera angle, encoding rate, and frame rate. The vehicle periodically sends location information to the MEC server. Then, the MEC server replies to the same vehicle with a warning message on road traffic collision avoidance. The warning message is generated in the MEC server based on the location information received from the vehicle and the image most recently received from the camera. In this paper, we define a flow from a vehicle (or camera) to the same vehicle (or camera) via an MEC server, not a flow from a camera to a vehicle via an MEC server, as a mission critical (MC) flow. We call a whole deadline of the MC flow the MC deadline. The MC deadline is defined as $100 \mathrm{~ms}$.

The key to improving the arrival ratio within the MC deadline rests on how to set each of the uplink and downlink deadlines. When deadline exceedance occurs in either the uplink or downlink radio section, eNB discards the exceeded packet even if does not exceed the MC deadline. This degrades the arrival ratio within the MC deadline. Therefore, it is important to adaptively set each deadline on the uplink and downlink radio sections.

El-Hajj et al. proposed an algorithm to decide the optimal deadlines of the uplink and downlink radio sections [23], [24] on the basis of the throughput in each link. By using the optimal deadline, the MAC scheduler can guarantee that the first packet of each flow arrives at the destination before the given deadline. However, they did not discuss the effect of heterogeneous traffic environment, namely, that chunk size on the radio uplink and downlink are not always the same on an MC flow. Therefore, we cannot apply this algorithm to our target system.

In this paper, to improve the arrival ratio within the $\mathrm{MC}$ deadline, we propose a deadline coordination function (DCF) for the DAS-QF that adaptively allocates uplink and downlink deadlines to each link in accordance with the MC deadline and the uplink and downlink congestion levels. Then, the DAS-QF with DCF decides priority in accordance with radio quality, chunk size, and allocated uplink and downlink deadlines. Simulation results show that the DAS-QF with DCF achieves a higher arrival ratio within the MC deadline than the DAS-QF on its own.

This paper is organized as follows. In Sect. 2, we describe cellular system and use case. We provide description of DAS-QF and its technical issue in Sect. 3. Section 4 introduces the DCF for improving the arrival ratio within the MC deadline for the MC flow. In Sect. 5, we evaluate the DCF using a computer simulation and discuss the results. We conclude in Sect. 6 with a brief summary.

\section{Cellular System and Use Case}

\subsection{Cellular System}

The cellular system is shown in Fig. 1, where it is assumed that a wide variety of UEs are connected to mobile networks. The UEs are divided into two types: group 1 and 2. Group 1 includes UEs that browse Web sites and streaming services; we call these the best effort UEs (BE-UEs). Group 2 includes UEs for road traffic collision avoidance services, such as connected cameras and vehicles; we call these the mission critical UEs (MC-UEs).

The MEC server plays the role of application server. A mission critical service such as road traffic collision avoidance is operated in the MEC server located in a nearby eNB to achieve low latency. The BE-UE accesses an application server on the Internet as usual to receive a certain desired service. In addition, the MEC server calculates each of the uplink and downlink deadlines and informs an eNB of the chunk size and the calculated deadlines.

The eNB has a MAC scheduling function and decides how to allocate radio resources to UEs. The DAS-QF that we use as a baseline for comparison allocates radio resources in accordance with the priority that is calculated on the basis of chunk size, radio quality, and uplink and downlink deadlines.

The home subscriber server (HSS) has a database for user identification and authentication. It also has a mapping table between a UE and a service that the UE utilizes and the QoS requirements defined in each service. The HSS has an

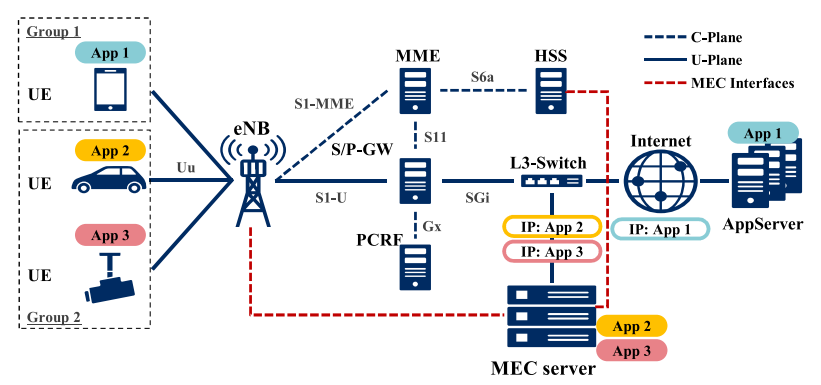

Fig. 1 Cellular system. 


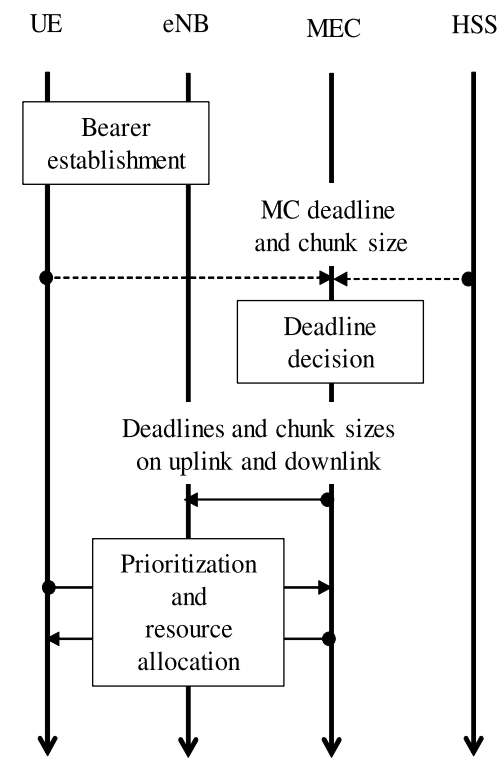

Fig. 2 Sequence of cellular system.

interface to inform the MEC server of the QoS requirements.

Other devices, such as the serving gateway and packet data network gateway (S/P-GW), mobility management entity (MME), and policy and charging rules function (PCRF), have various functions defined in the 3GPP specification.

The sequence of the cellular system is shown in Fig. 2. The cellular system first establishes a bearer between a UE and the eNB. The QoS requirements are defined on the basis of each application and contain the MC deadline. The MEC server receives information of the chunk size on uplink and the MC deadline from either the source UE or HSS when a new session is established or when the QoS requirements are changed in accordance with application mode switching. The HSS finds the QoS requirements by referencing the mapping table between a UE and a service that the UE utilizes. The MEC server decides the uplink and downlink deadlines and notifies the eNB of the uplink and downlink deadlines and each of the uplink and downlink chunk sizes. It is assumed that the MEC server can understand chunk size on the downlink, as it is created by the MEC server. The eNB calculates UE priority in accordance with each of the uplink and downlink deadlines and each of the uplink and downlink chunk sizes, and with the radio quality, and allocates radio resources to the UE on the basis of the calculated priority.

\subsection{Use Case}

This research assumes LTE vehicle-to-everything (V2X) services, such as vehicle-to-vehicle $(\mathrm{V} 2 \mathrm{~V})$, vehicle-topedestrian (V2P), and vehicle-to-infrastructure (V2I), via the MEC server. We assumed a country where driving lane is left side, such as Japan. We investigated an intersection reported in [25] where road traffic collisions often occur. Results showed that most road traffic collisions in Japan, where drivers keep to the left side of the road, are caused at intersections: right-turn collisions, rear-end collisions, and

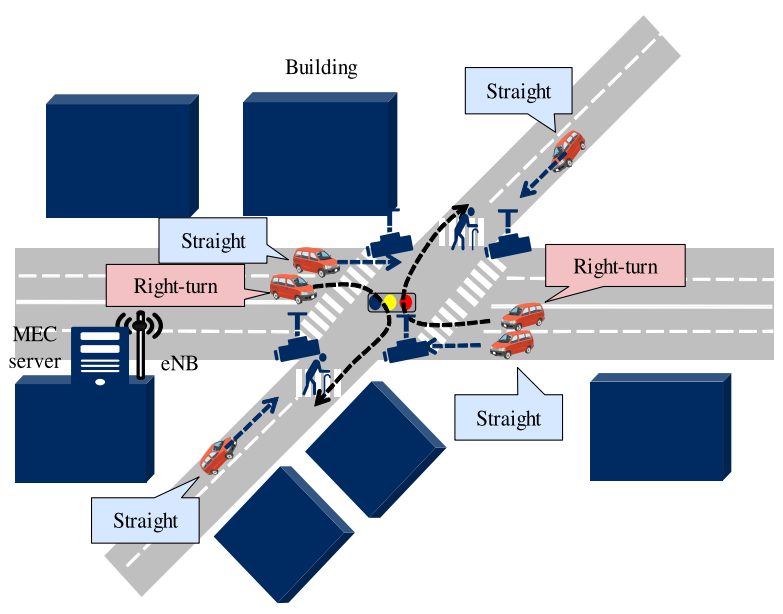

Fig. 3 Use case intersection.

so on. Right-turn collisions are particularly likely to occur at intersections that are bent sharply to the right, as shown in Fig. 3. It is noted that, in a country where drivers keep to the right side of the road, left-turn collisions are more dangerous than right-turn collisions.

In this paper, we advance discussions on the assumption of a country where drivers keep to the left side of the road. The use case examined in this paper is the intersection shown in Fig. 3. The camera periodically sends a monitoring image around the intersection to the MEC server, and the MEC server sends the camera a message for controlling the camera. Vehicles also send location information to the MEC server periodically. Then, the MEC server sends a warning message to the same vehicle on the basis of the monitoring image and location information.

\section{DAS-QF}

\subsection{MAC Scheduler}

The DAS-QF proposed in our earlier study [21] decides priority on the basis of radio quality, chunk size, and deadline to obtain a higher arrival ratio within the MC deadline. The DAS-QF locates emergency UEs by monitoring the progress of data transmission and preferentially gives transmission rights to the UE to avoid exceeding the deadline.

The concept of the DAS-QF is depicted in Fig. 4. The DAS-QF judges whether a chunk is an emergency or nonemergency by comparing the target throughput and the requested throughput. The target throughput is the average throughput needed to meet the deadline. The requested throughput is the throughput needed to meet the deadline at a certain point in time. If the requested throughput is higher than the target throughput, the DAS-QF categorizes the chunk state as emergency and sets the chunk priority to high so as to shorten the delay. Otherwise, the DAS-QF categorizes the chunk state as non-emergency and sets the chunk priority to low.

The detail of the DAS-QF is shown in Algorithm 1. 


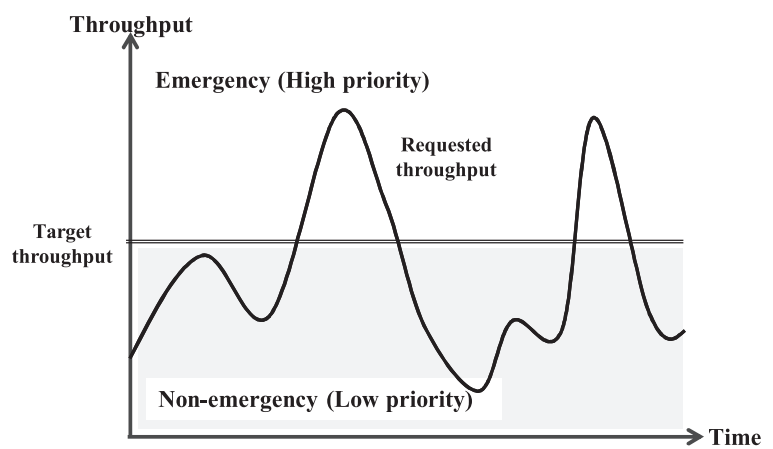

Fig. 4 Relation between chunk states and priority.

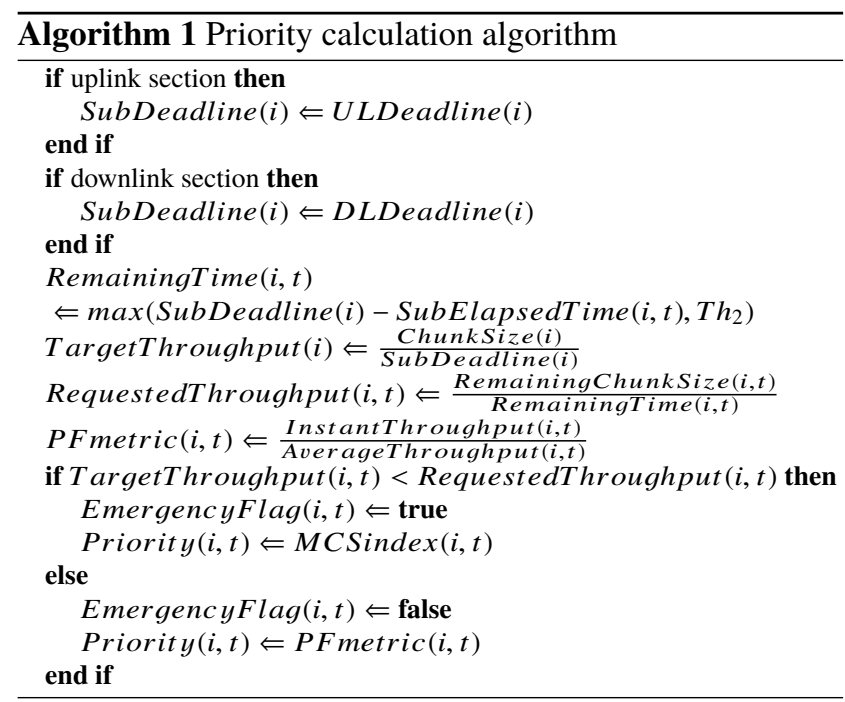

Algorithm 1 outputs the chunk state, i.e., emergency or non-emergency, and chunk priority. The parameters are determined based on the inputs of Algorithm 1, i.e., the deadline, elapsed time, chunk size, instant throughput, average throughput, and radio quality index. Table 1 lists the parameters of Algorithm 1 for chunk $i$ at the $t$-th TTI. The priority calculation is executed each transmission time interval (TTI). $T h_{2}$ is used to avoid RemainingTime $(i, t)$ being equal to zero or a negative value. When RemainingTime $(i, t)$ is zero, RequestedThroughput $(i, t)$ is indeterminate. RequestedThroughput $(i, t)$ is a negative value when RemainingTime $(i, t)$ is a negative value.

If the RequestedThroughput $(i, t)$ is higher than the TargetThroughput(i), we set the chunk state as "emergency" because it is not likely to meet the deadline. The DAS-QF also considers radio quality in addition to the emergency degree in order to efficiently use radio resources, which are finite. That is, the better the radio quality, the higher the priority. We use the modulation and coding scheme (MCS) as a radio quality index. If RequestedThroughput $(i, t)$ is lower than TargetThroughput(i), we set the chunk state as "nonemergency" because the chunk is making good progress. Then, the DAS-QF decides the priority of the chunk by
Table 1 Algorithm parameters for chunk $i$ on $t$-th TTI.

\begin{tabular}{|c|c|}
\hline Parameter & Explanation \\
\hline TargetThroughput(i) & Target throughput \\
\hline ChunkSize(i) & Chunk size \\
\hline SubDeadline $(i)$ & Uplink or downlink deadline \\
\hline ULDeadline $(i)$ & Uplink deadline \\
\hline DLDeadline $(i)$ & Downlink deadline \\
\hline SubElapsedTime $(i)$ & $\begin{array}{l}\text { Elapsed time on uplink or down- } \\
\text { link section }\end{array}$ \\
\hline RequestedThroughput $(i, t)$ & Requested throughput \\
\hline RemainingChunkSize $(i, t)$ & Remaining chunk size \\
\hline RemainingTime $(i, t)$ & Remaining time to deadline \\
\hline PFmetric $(i, t)$ & $\begin{array}{l}\text { Priority of chunk } i \text { that has no } \\
\text { emergency flag }\end{array}$ \\
\hline InstantThroughput $(i, t)$ & $\begin{array}{l}\text { Instant throughput of UE that has } \\
\text { chunk } i\end{array}$ \\
\hline AverageThroughput $(i, t)$ & $\begin{array}{l}\text { Average throughput of UE that } \\
\text { has chunk } i\end{array}$ \\
\hline EmergencyFlag $(i, t)$ & Flag to show degree of urgency \\
\hline MCSindex $(i, t)$ & $\begin{array}{l}\text { MCS index of UE that has chunk } \\
i\end{array}$ \\
\hline $\operatorname{Priority}(i, t)$ & Priority \\
\hline
\end{tabular}

means of a PFmetric that considers the fairness of the transmission right. PFmetric is defined by a fraction of the average throughput and instant throughput.

After calculating the priority of each chunk, the DAS$\mathrm{QF}$ allocates radio resources to each chunk on the basis of its priority. Radio resource allocation assigns radio resources to the UE that has the best radio quality among all the emergency UEs. If radio resources do not need to be allocated to emergency UEs, the radio resource allocation assigns them to non-emergency UEs in accordance with each priority PFmetric.

\subsection{Technical Issue of DAS-QF}

The DAS-QF allocates the same deadline to all UEs. In Fig. 5, a vehicle and a camera connect to the same eNB. It is assumed that the chunk sizes of the vehicle and camera are light and heavy, respectively. In this case, the effective uplink delay of the camera chunk is likely to be larger than that of the vehicle chunk. Even so, the DAS-QF allocates the same deadline $(\alpha)$, as shown in Fig. 5, to both vehicle and camera. When the effective uplink delay of a chunk exceeds the uplink deadline, the chunk is discarded in the eNB. In Fig. 5, the effective uplink delays of the vehicle and camera are $\beta_{v}(<\alpha)$ and $\beta_{c}(>\alpha)$, respectively. Then, the chunk from the vehicle to the MEC server is not discarded in the eNB, while on the other hand, the chunk from the camera to the MEC server is discarded. As a result, the DAS-QF causes a degradation of the arrival ratio within the MC deadline for cameras. 


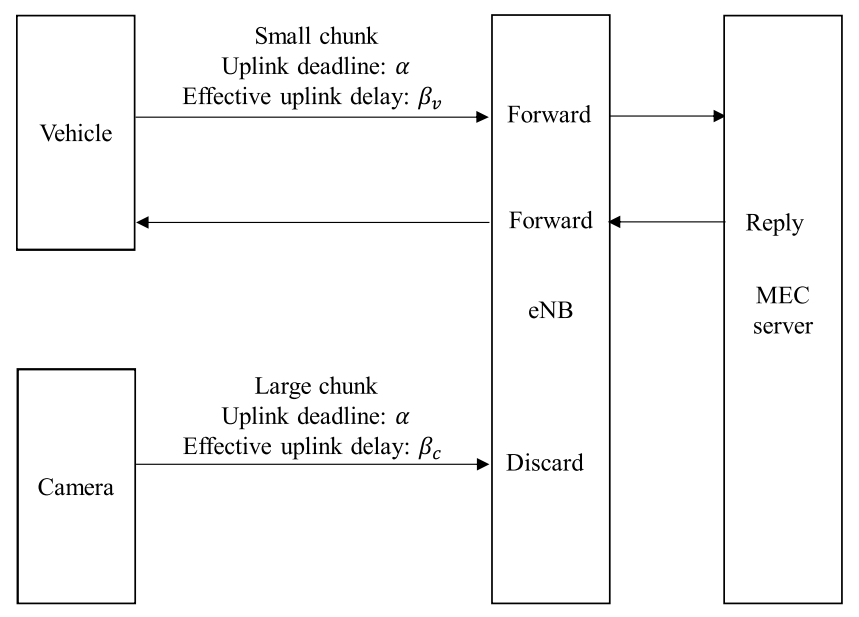

Fig.5 Technical issue of DAS-QF.

\section{DCF}

We propose a DCF that decides the uplink and downlink deadlines input to an eNB. The DCF consist of three steps, which are shown in Fig. 6. As the first step, the DCF calculates the minimum coordination amount needed for satisfying the arrival ratio within the MC deadline. It calculates the uplink and downlink congestion levels as the second step. As the third step, the DCF updates each of the uplink and downlink deadlines in accordance with the minimum coordination amount and the uplink and downlink congestion levels.

\subsection{Step 1: Calculation of Minimum Coordination Amount}

It is assumed that the number of chunks of MC flow $f$ measured at a certain interval is $n$. We define $n$ chunks sorted in chronological order as $x_{1}, x_{2}, \cdots, x_{k}, \cdots, x_{n}$, and the range of $k$ is $1 \leq k \leq n$. The MC deadline vector $\boldsymbol{a}_{f x}$ is expressed as

$$
\boldsymbol{a}_{f x}=\left(a_{x_{1}}, a_{x_{2}}, \cdots, a_{x_{k}}, \cdots, a_{x_{n}}\right),
$$

where $a_{x_{k}}$ is the MC deadline of chunk $x_{k}$. The effective $\mathrm{MC}$ delay vector $\boldsymbol{b}_{f x}$ is expressed as

$$
\boldsymbol{b}_{f x}=\left(b_{x_{1}}, b_{x_{2}}, \cdots, b_{x_{k}}, \cdots, b_{x_{n}}\right) \text {, }
$$

where $b_{x_{k}}$ is the effective MC delay of chunk $x_{k}$. It is assumed that UE embeds a unique identifier (ID) into a message and records the time when the UE sends the message to the MEC server. When the MEC server sends a reply message to the same UE, the MEC server also embeds the received unique ID into the reply message. The UE can measure effective MC delay based on the time when the message was sent to the MEC server, the time when the message was received from the MEC server, and unique ID. The UE advertises effective MC delay to the MEC server.

The remaining time vector $\boldsymbol{\delta}_{f x}$ is expressed as

$$
\boldsymbol{\delta}_{f x}=\left(\delta_{x_{1}}, \delta_{x_{2}}, \cdots, \delta_{x_{k}}, \cdots, \delta_{x_{n}}\right) \text {, }
$$

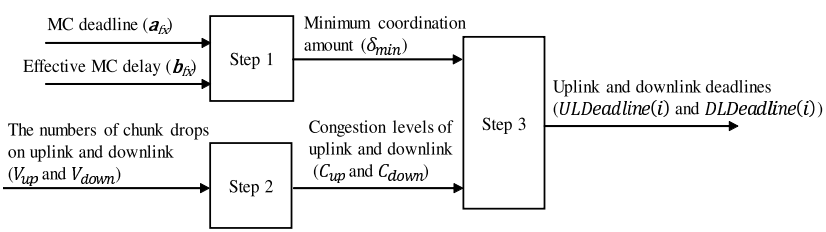

Fig. 6 Three steps of DCF.

where $\delta_{x_{k}}$ is the remaining time to the MC deadline of chunk $x_{k}$. The remaining time vector $\boldsymbol{\delta}_{f x}$ is formulated as

$$
\boldsymbol{\delta}_{f x}=\boldsymbol{a}_{f x}-\boldsymbol{b}_{f x} .
$$

The DCF sorts $x_{1}, x_{2}, \cdots, x_{k}, \cdots, x_{n}$ in descending order of the remaining time to the MC deadline, and sorted chunks are $y_{1}, y_{2}, \cdots, y_{k}, \cdots, y_{n}$. The sorted remaining time vector $\boldsymbol{\delta}_{f y}$ is expressed as

$$
\boldsymbol{\delta}_{f y}=\left(\delta_{y_{1}}, \delta_{y_{2}}, \cdots, \delta_{y_{k}}, \cdots, \delta_{y_{n}}\right),
$$

where $\delta_{y_{k}}$ is the remaining time to the MC deadline of chunk $y_{k}$. Then, minimal coordination amount $\delta_{\min }$ is expressed as

$$
\delta_{\min }=\delta_{y_{\left\lceil n \cdot Q_{f}\right\rceil}},
$$

where $Q_{f}$ is the required arrival ratio within the MC deadline of flow $f$, and the range of $Q_{f}$ is $0<Q_{f} \leq 1$. When $\delta_{\text {min }}$ is equal to zero or a positive value, the MC flow $f$ satisfies the required arrival ratio within the $\mathrm{MC}$ deadline. Therefore, Step 2 and 3 are not executed when $\delta_{\min }$ is equal to zero or a positive value. In contrast, when $\delta_{\min }$ is a negative value, the MC flow $f$ does not satisfy the required arrival ratio within the MC deadline. To satisfy the required arrival ratio within the MC deadline, step 2 and 3 are executed when $\delta_{\min }$ is a negative value.

\subsection{Step 2: Estimation of Congestion Level}

In this step, the DCF estimates the uplink and downlink congestion levels in accordance with the number of chunk drops in the uplink and downlink. The uplink congestion level $C_{u p}$ is formulated as

$$
C_{u p}=\frac{V_{u p}}{V_{u p}+V_{\text {down }}},
$$

where $V_{u p}$ and $V_{\text {down }}$ are the number of chunk drops at uplink and at downlink, respectively. The downlink congestion level $C_{\text {down }}$ is formulated as

$$
C_{\text {down }}=\frac{V_{\text {down }}}{V_{u p}+V_{\text {down }}} .
$$

$V_{u p}$ and $V_{\text {down }}$ are measured by the eNB, and the eNB advertises $V_{u p}$ and $V_{\text {down }}$ to the MEC server. We assume that the time stamp is used for the measurement of $V_{u p}$ and $V_{\text {down }}$, and all clocks of UEs, eNBs and the MEC server are synchronized. An UE embeds the time stamp into a chunk when 
the UE sends the chunk to the MEC server, and the MEC server embeds another time stamp into the chunk when the MEC server sends the chunk to the UE. The eNB calculates the remaining time to the deadline by using the time stamp and deadline when the eNB receives the chunk. The eNB discards the chunk and increase the drop count when the chunk does not meet the deadline.

When the number of chunk drops is high, it is assumed that the congestion level is high compared to when the number of chunk drops is low.

\subsection{Step 3: Update of Uplink and Downlink Deadlines}

This step updates the uplink and downlink deadlines of chunk $i$ in accordance with the minimum coordination amount $\delta_{\text {min }}$ and uplink and downlink congestion levels $C_{u p}$ and $C_{\text {down }}$. The uplink and downlink deadlines of chunk $i$ are ULDeadline $(i)$ and DLDeadline $(i)$, respectively and are defined as (9) and (10), respectively.

$$
\operatorname{ULDeadline}(i)=\delta_{\text {min }} \cdot C_{\text {down }}+\operatorname{ULDeadline}(i-1)
$$

$$
\text { DLDeadline }(i)=\delta_{\text {min }} \cdot C_{u p}+\operatorname{DLDeadline}(i-1)
$$

The uplink and downlink deadlines of the chunk whose effective MC delay exceeds the MC deadline are set to a smaller value in the range that is not generating chunk drops in the eNB in order to raise the priority of the chunk. A negative $\delta_{\min }$ means that the previous chunk $i-1$ could not been delivered within the MC deadline. The next chunk $i$ also cannot meet the MC deadline in the same network conditions as the previous chunk. Therefore, the sum of the next uplink and downlink deadlines is set to a shorter value than the sum of the previous uplink and downlink deadlines. This results in a shorter effective MC delay of the next chunk than that of the previous chunk because the next chunk has priority use of radio resources according to Algorithm 1. By this step, it is expected that the next chunk meets the MC deadline.

After step 3, the MEC server sends ULDeadline $(i)$ and DLDeadline $(i)$ to the eNB that the UE is connected to. The eNB then decides the UE priority on the basis of the radio quality, chunk size, and target deadline information received from the MEC server, and allocates radio resources to the UE on the basis of this priority.

\section{Performance Evaluation}

We investigated the effect of the DCF through simulations using ns-3, a general network simulation framework that is widely used in network research [26]. Specifically, we compared the performance of the DAS-QF with DCF to that with the DAS-QF on its own. In [21], it has been shown that the DAS-QF is more effective than the other conventional schedulers, i.e., the PayDA and proportional fair.
Table 2 Simulation parameters for eNB.

\begin{tabular}{|c|c|}
\hline Number of eNBs & $7(1$ cell per eNB $)$ \\
\hline Radius & $289 \mathrm{~m}$ (distance between eNBs: $500 \mathrm{~m})$ \\
\hline Uplink and downlink frequency & $2 \mathrm{GHz}$ \\
\hline Antenna height & $32 \mathrm{~m}$ \\
\hline Uplink and dlownlink bandwidth & $20 \mathrm{MHz}(=100$ resource blocks $)$ \\
\hline Fading & Pedestrian A \\
\hline
\end{tabular}

\subsection{Simulation Environment}

We investigated an intersection where road traffic collisions often occur in order to evaluate the DCF in a realistic environment. Specifically, we created the realistic environment shown in Fig. 3 on ns-3. The simulation of urban mobility (SUMO) [27], [28] was used to define the mobility model of vehicles to simulate realistic mobility behavior of vehicles.

In this work, we evaluate the DAS-QF with DCF under the LTE assumption, but it is not limited to the LTE. For example, we expect that the 5 th generation $(5 \mathrm{G})$ mobile network will improve the arrival ratio within the MC deadline compared to LTE because the network latency of $5 \mathrm{G}$ will be smaller. We also expect the DAS-QF with DCF to be effective in $5 \mathrm{G}$ because the network may be congested by increasing the number of connected devices.

The simulation parameters of the eNB are listed in Table 2 . The cell formation is seven hexagonal cells, with one eNB allocated at the center of each cell. The road environment is allocated around the intersection on the center cell, which has one eNB and several buildings for interference. The six neighboring cells are also for interference. We measure the performance only on the center cell. It is assumed that new vehicle appears in the center cell at the same time as a vehicle moves out of the center cell range so that we keep the number of vehicle and network load in the center cell constant. Therefore, this simulation does not have handover situation. When the new vehicle appears in the center cell, it is assumed that the new vehicle sends a chunk after bearer establishment by the initial attach procedure. The effective MC delay does not include the delay for bearer establishment. The uplink and downlink frequency and bandwidth are $2 \mathrm{GHz}$ and $20 \mathrm{MHz}$ used in LTE, respectively. Pedestrian A [29] is used as the fading model.

Table 3 lists the simulation parameters of the UEs. MCUEs, which are delay-sensitive, are vehicles and cameras, and BE-UEs, which are delay-non-sensitive, are pedestrians. The BE-UE accesses an application server on the Internet as usual to receive a certain desired service. The BE-UE sends a chunk to the application server. The application server replies a chunk for the desired service to the BE-UE. We call a flow from a BE-UE to the same BE-UE via an application server the BE flow. The effective delay of the BE flow is defined as the effective BE delay. The number of cameras is 4 , assuming that each camera is located around each corner of the intersection. The first chunk of each vehicle or camera or pedestrian is randomly generated between 0 and $100 \mathrm{~ms}$ of 
Table 3 Simulation parameters for UEs.

\begin{tabular}{|c|c|}
\hline Tx power & $23 \mathrm{dBm}$ \\
\hline Uplink power control & Enabled \\
\hline Antenna height & $1.5 \mathrm{~m}$ \\
\hline Number of vehicles & 100 \\
\hline Number of cameras & 4 \\
\hline Number of pedestrians & 100 \\
\hline Chunk size of vehicle in uplink and downlink & $1.7 \mathrm{~KB}$ \\
\hline Chunk size of camera in uplink & $37.5 \mathrm{~KB}$ \\
\hline Chunk size of camera in downlink & $1.7 \mathrm{~KB}$ \\
\hline Chunk size of pedestrian in uplink and downlink & $1.7 \mathrm{~KB}$ \\
\hline Traffic generation interval & $100 \mathrm{~ms}$ \\
\hline MC deadline & $100 \mathrm{~ms}$ \\
\hline
\end{tabular}

simulation time [30]. Next chunks are generated at 100-ms intervals until the end of simulation.

We investigated a proper bitrate of video traffic with the Toomer that is a video of a certain intersection and is provided in [31]. We set the frame rate, resolution, and codec to $10 \mathrm{fps}, 1280 * 720$ (HD 720), and H.264, respectively. We detected cars and persons with the YOLOv3 [32] and measured the mean average precision (mAP) for cars and persons. When the video was transcoded to $3 \mathrm{Mbps}$, the mAP of $95 \%$ frames in all the frames was more than 0.9. Assuming that the mAP of $95 \%$ frames in all the frames was more than 0.9 is practically enough accuracy, the video traffic was set to $3 \mathrm{Mbps}$. Since each camera sent an image to the MEC server every $100 \mathrm{~ms}$, the size of each image data chunk was $37.5 \mathrm{~KB}$. In the $3 \mathrm{GPP}$ specification, codec delay is not included in the deadline. Therefore, this paper does not include the codec delay in the effective MC delay.

The sizes of other chunks (such as location information from a vehicle, messages for warning and control from the MEC server, and a message that a pedestrian sends and receive) are all $1.7 \mathrm{~KB}$. The size of the chunk from the vehicle to the MEC server and the size of the chunk from the MEC server to the vehicle was also the same. Therefore, in the DAS-QF, the uplink and downlink deadlines were set to $50 \mathrm{~ms}$ and $50 \mathrm{~ms}$, respectively. $T h_{2}$ was set to $1 \mathrm{~ms}$.

The user datagram protocol (UDP) is used as a transport protocol to exchange chunks, as 3GPP stipulates that V2X such as connected car services use UDP [33]. The maximum transmission $(\mathrm{Tx})$ power is $23 \mathrm{dBm}$, and the power control specified in [34] is applied to the simulation environment.

In the paper, it was assumed that the delay for the sequence of updating uplink and downlink deadlines was negligible because the MEC server was located nearby the eNB and notified the eNB of the updated deadlines by using a control plane, whose congestion level is lower than that of a data plane.

\subsection{Simulation Results}

The QoS requirements for connected car services in terms of the MC deadline and arrival ratio within the MC deadline

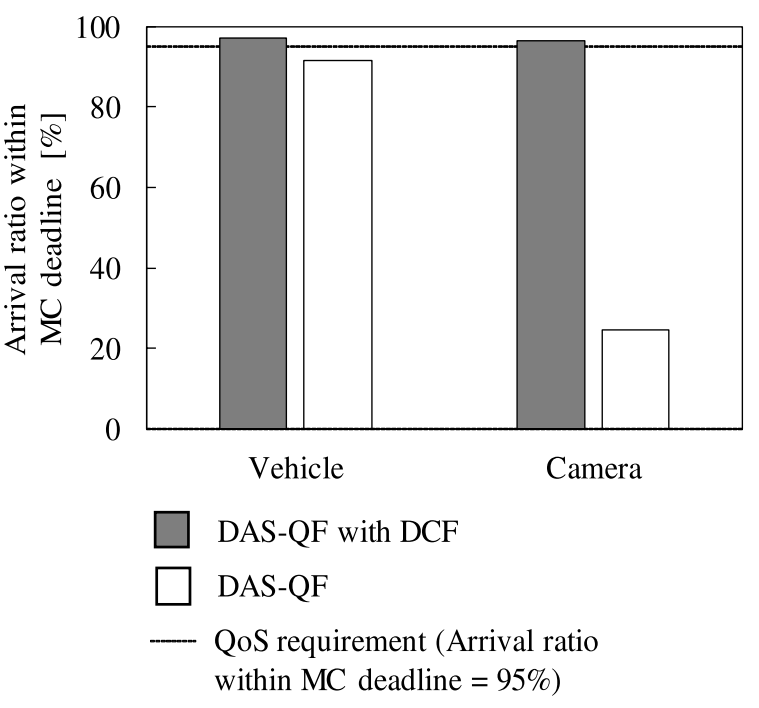

Fig. 7 Arrival ratio within MC deadline.

have been presented in [9]. According to [9], in the case of urban intersections, the $\mathrm{MC}$ deadline and the arrival ratio within the MC deadline are $100 \mathrm{~ms}$ and $95 \%$, respectively. We define these here as network requirements for connected car services.

Figure 7 shows the arrival ratio within the MC deadline, which is formulated as

$$
P_{c}=\frac{M_{b}}{M_{a}}
$$

where $M_{a}$ is the total number of chunks that MC-UEs sent within the simulation time and $M_{b}$ is the number of chunks that MC-UEs received before the MC deadline within the simulation time.

As shown in 7, the DAS-QF with DCF obtained higher vehicle and camera arrival ratios within the MC deadlines than the DAS-QF on its own. Specifically, the DAS-QF with DCF could attain $95 \%$ of the arrival ratio within the MC deadline at both vehicle and camera, while the DAS-QF could not.

For the vehicle arrival ratio within the MC deadline, the DAS-QF also obtained a high arrival ratio within the MC deadline compared to the camera arrival ratio within the MC deadline. In this paper, the size of the chunk from the vehicle to the MEC server and the size of the chunk from the MEC server to the vehicle was also the same. Therefore, the DAS-QF, where each of the uplink and downlink deadlines was set to the same value, i.e., $50 \mathrm{~ms}$, worked well.

For the camera arrival ratio within the MC deadline, the DAS-QF could not obtain a high arrival ratio because it allocated the uplink and downlink deadlines the same value, i.e., $50 \mathrm{~ms}$, even though the size of the chunk from the camera to the MEC server was larger than that of the chunk from the MEC server to the camera.

The reason the DAS-QF with DCF obtained a higher success ratio than the DAS-QF on its own was that it could adaptively set each of the uplink and downlink deadlines, 


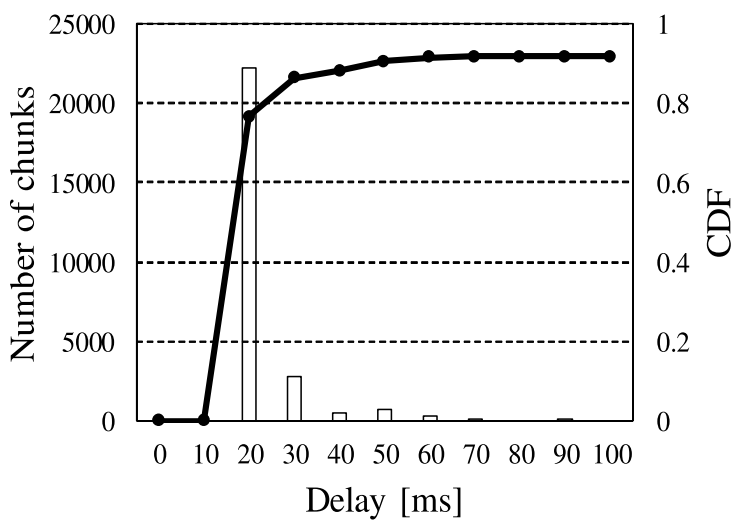

Fig. 8 Delay distribution of DAS-QF on its own (Vehicle).

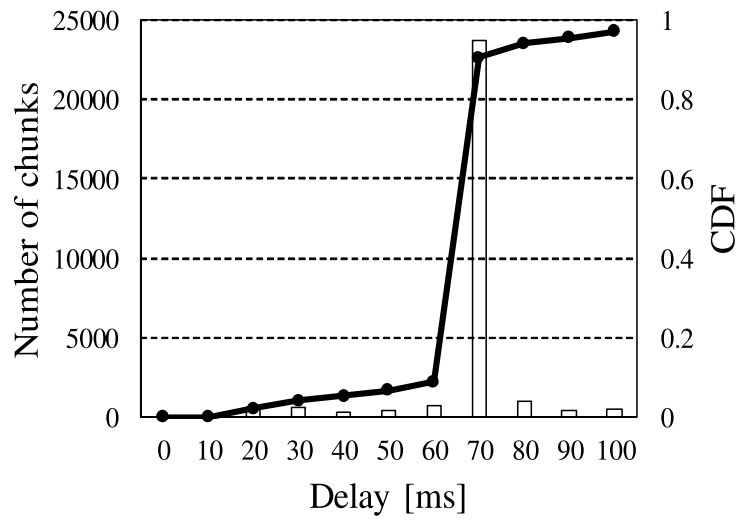

Fig. 9 Delay distribution of DAS-QF with DCF (Vehicle).

and thus decreased the number of chunk drops. Figures 811 show the delay distribution of chunks and its cumulative distribution function (CDF) for each case. The horizontal axis is the delay of each chunk, e.g., $30 \mathrm{~ms}$ on the horizontal axis means $20 \mathrm{~ms}<$ delay $\leq 30 \mathrm{~ms}$.

In the case of vehicles, the number of chunks that did not meet the MC deadline on the DAS-QF with DCF was approximately one third of that of the chunks on the DASQF, as shown by the comparison of Figs. 8 and 9. In the case of cameras, the number of chunks that did not meet the MC deadline on the DAS-QF with DCF was approximately one fifteenth of that of the chunks on the DAS-QF, as shown by the comparison of Figs. 10 and 11.

The peak of delay distribution for vehicles existed at the delay of $20 \mathrm{~ms}$ in Fig. 8 when the DCF was not used. In contrast, when the DCF was used, the peak shifted from $20 \mathrm{~ms}$ to $70 \mathrm{~ms}$ in Fig. 9. This shift generated vacant radio resources that were then efficiently utilized by assigning them to camera traffic. The DAS-QF with DCF shifted radio resources that are likely to be assigned to vehicles to cameras by setting the deadline of camera to a smaller value. From Figs. 10 and 11, we can confirm that the vacant radio resources were assigned to camera traffic. Approximately 600 chunks did not meet the MC deadline (100 ms) in Fig. 10. In

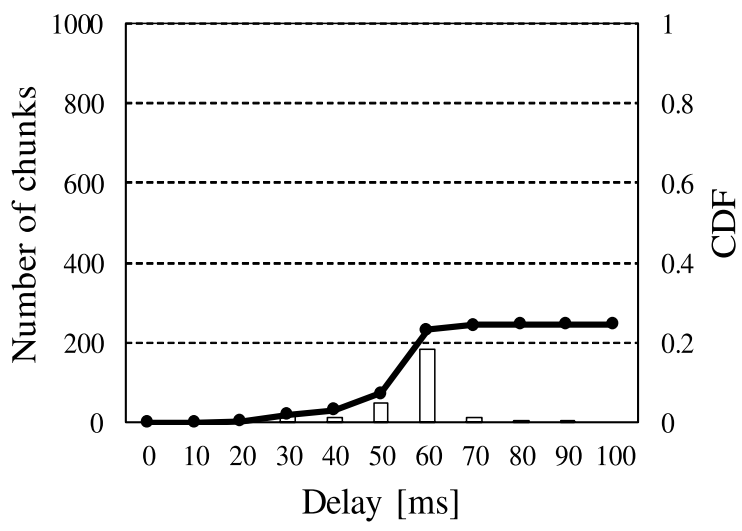

Fig. 10 Delay distribution of DAS-QF on its own (Camera).

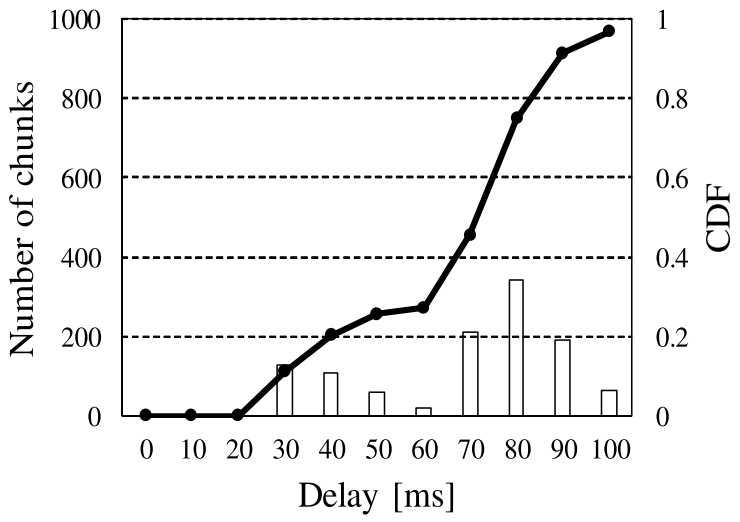

Fig. 11 Delay distribution of DAS-QF with DCF (Camera).

Fig. 11, approximately $93 \%$ of these chunks were distributed to the left of $100 \mathrm{~ms}$ on the horizontal axis. These results demonstrate that the DAS-QF with DCF could obtain higher arrival ratios within the $\mathrm{MC}$ deadlines for both vehicles and cameras than the DAS-QF on its own.

The impact on BE flow is discussed. The DCF degraded the arrival rate for the BE flow and average effective BE delay compared to the DAS-QF on its own. The arrival rates for the BE flow of the DAS-QF with DCF and DAS-QF on its own were 0.88 and 0.90 , respectively. The average effective BE delays of the DAS-QF with DCF and DAS-QF on its own were $254 \mathrm{~ms}$ and $245 \mathrm{~ms}$, respectively. This is because the DAS-QF with DCF allocated less amount of radio resources to the BE flow than the DAS-QF on its own. Actually, the total bitrates received by all the BE-UEs for the DAS-QF with DCF and the DAS-QF on its own were $12.5 \mathrm{Mbps}$ and 13.0 Mbps, respectively.

\section{Conclusion}

In this paper, we have proposed a DCF that adaptively allocates uplink and downlink deadlines for MC flows. The proposed DCF calculates the minimum coordination amount needed for satisfying the arrival ratio within the MC deadline 
and the uplink and downlink congestion levels. It updates the uplink and downlink deadlines on the basis of their calculation results in order to avoid unintentional drops in the eNB.

We investigated the DCF in a realistic environment by simulating an intersection where road traffic collisions often occur on ns-3 and then evaluating the performance. Results showed that the DAS-QF with DCF achieved a higher performance than the DAS-QF on its own. Specifically, the vehicle arrival ratio within the MC deadline of the DASQF with DCF achieved an improvement of approximately $5 \%$ compared to the DAS-QF. In addition, the camera arrival ratio within the $\mathrm{MC}$ deadline of the DAS-QF with DCF achieved an improvement of approximately $70 \%$ compared to the DAS-QF. The QoS requirements for connected car services were achieved through this improvement by applying the DCF to the DAS-QF.

\section{Acknowledgments}

This paper includes some of the results from the "Research and development project for efficient and optimized use of radio resource in mobile networks with a variety of IoT devices" commissioned by the Ministry of Internal Affairs and Communications, Japan.

\section{References}

[1] Global status report on road safety 2015, World Health Organization, 2015.

[2] ITARDA, http://www.itarda.or.jp/english/

[3] M. Xie, Y. Shang, Z. Yang, Y. Jing, and H. Zhou, "A novel MBSFN scheme for vehicle-to-vehicle safety communication based on LTE network," 2015 IEEE 82nd Vehicular Technology Conference (VTC2015-Fall), pp.1-5, IEEE, 2015.

[4] S. Chen, J. Hu, Y. Shi, and L. Zhao, "LTE-V: A TD-LTE-based v2x solution for future vehicular network," IEEE Internet Things J., vol.3, no.6, pp.997-1005, 2016.

[5] S.h. Sun, J.1. Hu, Y. Peng, X.M. Pan, L. Zhao, and J.y. Fang, "Support for vehicle-to-everything services based on lte," IEEE Wireless Commun., vol.23, no.3, pp.4-8, 2016.

[6] S. Vishnu, U. Ramanadhan, N. Vasudevan, and A. Ramachandran, "Vehicular collision avoidance using video processing and vehicleto-infrastructure communication," 2015 International Conference on Connected Vehicles and Expo (ICCVE), pp.387-388, IEEE, 2015.

[7] J. Temperton, "One nation under CCTV: The future of automated surveillance," Aug. 2015.

[8] F. Langfitt, "In China, beware: A camera may be watching you," Jan. 2013.

[9] 3GPP, "Study on LTE support for vehicle to everything (V2X) services," Technical Report, TR 22.885, 2015.

[10] T. Iwai, D. Kominami, M. Murata, R. Kubo, and K. Satoda, "Mobile network architectures and context-aware network control technology in the IoT era," IEICE Trans. Commun., vol.E101-B, no.10, pp.20832093, Oct. 2018.

[11] K. Zhang, Y. Mao, S. Leng, Q. Zhao, L. Li, X. Peng, L. Pan, S. Maharjan, and Y. Zhang, "Energy-efficient offloading for mobile edge computing in 5G heterogeneous networks," IEEE Access, vol.4, pp.5896-5907, 2016.

[12] T. Taleb, K. Samdanis, B. Mada, H. Flinck, S. Dutta, and D. Sabella, "On multi-access edge computing: A survey of the emerging $5 \mathrm{G}$ network edge cloud architecture and orchestration," IEEE Commun.
Surveys Tutss, vol.19, no.3, pp.1657-1681, 2017.

[13] P. Porambage, J. Okwuibe, M. Liyanage, M. Ylianttila, and T. Taleb, "Survey on multi-access edge computing for internet of things realization," IEEE Commun. Surveys Tuts., vol.20, no.4, pp.2961-2991, 2018.

[14] Y. Ai, M. Peng, and K. Zhang, "Edge computing technologies for internet of things: A primer," Digital Communications and Networks, vol.4, no.2, pp.77-86, 2018.

[15] C.V. Networking, "Cisco visual networking index: Global mobile data traffic forecast update, 2017-2022 white paper," 2016.

[16] A. Nikravesh, D.R. Choffnes, E. Katz-Bassett, Z.M. Mao, and M. Welsh, "Mobile network performance from user devices: A longitudinal, multidimensional analysis," International Conference on Passive and Active Network Measurement, pp.12-22, Springer, 2014.

[17] C.L. Liu and J.W. Layland, "Scheduling algorithms for multiprogramming in a hard-real-time environment," J. ACM, vol.20, no.1, pp.46-61, Jan. 1973.

[18] M. Haferkamp, B. Sliwa, C. Ide, and C. Wietfeld, "Payload-size and deadline-aware scheduling for time-critical cyber physical systems," 2017 Wireless Days, IEEE, March 2017

[19] M.M. Andreozzi, G. Stea, A. Bacioccola, and R. Rossi, "Flexible scheduling for real-time services in high-speed packet access cellular networks," 2009 European Wireless Conference, IEEE, May 2009.

[20] K.M. Elsayed and A.K. Khattab, "Channel-aware earliest deadline due fair scheduling for wireless multimedia networks," Wireless Pers. Commun., vol.38, no.2, pp.233-252, 2006.

[21] N. Itoh, M. Morita, T. Iwai, K. Satoda, and R. Kubo, "A deadlineaware scheduling scheme for connected car services using mobile networks with quality fluctuation," IEICE Trans. Commun., vol.E102-B, no.3, pp.474-483, March 2019.

[22] N. Itoh, H. Kaneko, A. Kohiga, T. Iwai, and H. Shimonishi, "Novel packet scheduling for supporting various real-time IoT applications in LTE networks," 2017 IEEE International Workshop Technical Committee on Communications Quality and Reliability (CQR), IEEE, May 2017.

[23] A.M. El-Hajj and Z. Dawy, "On delay-aware joint uplink/downlink resource allocation in OFDMA networks," 2013 IEEE Symposium on Computers and Communications (ISCC), pp.000257-000262, IEEE, 2013.

[24] A.M. El-Hajj, Z. Dawy, and W. Saad, "A stable matching game for joint uplink/downlink resource allocation in OFDMA wireless networks," 2012 IEEE International Conference on Communications (ICC), pp.5354-5359, IEEE, 2012

[25] SONPO, http://www.sonpo.or.jp/efforts/reduction/

[26] S. Siraj, A.K. Gupta, and Rinku-Badgujar, "Network simulation tools survey," International J. Advanced Research in Computer and Communication Engineering, vol.1, no.4, pp.201-210, June 2012.

[27] M. Behrisch, L. Bieker, J. Erdmann, and D. Krajzewicz, "SUMOSimulation of urban mobility," Proc. Third International Conference on Advances in System Simulation, 2011.

[28] M. Behrisch, D. Krajzewicz, and M. Weber, eds., Simulation of Urban Mobility, Springer Berlin Heidelberg, 2014.

[29] G. Piro, N. Baldo, and M. Miozzo, "An LTE module for the ns-3 network simulator," Proc. 4th International ICST Conference on Simulation Tools and Techniques, SIMUTools'11, pp.415-422, ICST, Brussels, Belgium, Belgium, ICST (Institute for Computer Sciences, Social-Informatics and Telecommunications Engineering), 2011.

[30] 3GPP, "Service requirements for v2x services," Technical Report, TS $22.185,2017$

[31] R.T. Mullapudi, S. Chen, K. Zhang, D. Ramanan, and K. Fatahalian, "Online model distillation for efficient video inference," Proc. IEEE International Conference on Computer Vision, pp.3573-3582, 2019

[32] J. Redmon and A. Farhadi, "YOLOv3: An incremental improvement," arXiv: 1804.02767, 2018

[33] 3GPP, "User equipment (UE) to V2X control function," Technical Report, TS 24.386, 2017. 
[34] 3GPP, "Physical layer procedures (release 10)," Technical Report, TS 36.213, 2011.

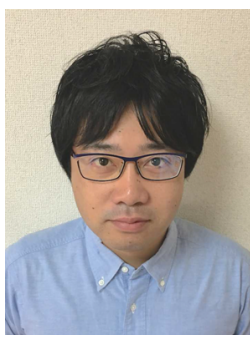

Nobuhiko Itoh received his B.E. and M.E. degrees in electrical engineering from Kansai University, Japan, in 2006 and 2008. In 2008, he joined NEC Corporation, Japan, and is now a senior researcher at System Platform Research Laboratories. He is currently pursuing his Ph.D. in integrated design engineering at Keio University, Japan. His research interests include system control, mobile networks, and IoT service networks. He received the Best Paper Award at the IEEE CQR Workshop 2017.

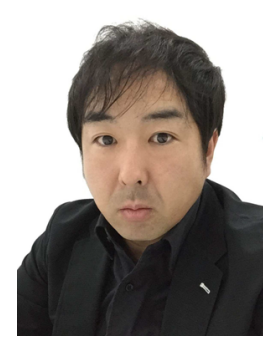

Takanori Iwai received his B.E. and M.E. degrees in electrical and electronic engineering from Shinshu University, Japan, in 2002 and 2004. In 2004, he joined NEC Corporation, Japan, and is now a principal researcher at System Platform Research Laboratories. He is currently pursuing his Ph.D. in integrated design engineering at Keio University, Japan. His research interests include system control, mobile and wireless networking, and IoT service networks. He received the Electrical Science and Engineering Award from the Promotion Foundation for Electrical Science and Engineering in 2016.

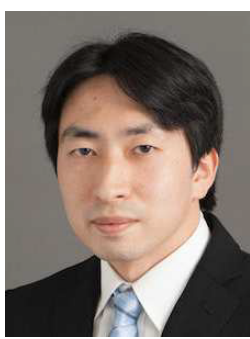

Ryogo Kubo received his B.E. degree in system design engineering and his M.E. and Ph.D. degrees in integrated design engineering from Keio University, Japan, in 2005, 2007, and 2009. In 2007, he joined the NTT Access Network Service Systems Laboratories, NTT Corporation, Japan. Since 2010, he has been with Keio University, Japan, where he is currently an Associate Professor at the Department of Electronics and Electrical Engineering. From 2019 to 2020, he also held the position of Honorary Research Fellow at the Department of Electronic and Electrical Engineering, University College London (UCL), London, UK. His research interests include system control, optical communications, networking, and cyber-physical systems. He received the Best Paper Award from the IEICE Communications Society in 2011, the IEEE International Conference on Communications (ICC '12) Best Paper Award in 2012, the Leonard G. Abraham Prize from the IEEE Communications Society in 2013, and the 2018 IEEE International Conference on Intelligence and Safety for Robotics (ISR '18) Best Paper Award in 2018. 